\title{
Growing Plants in Space: Manipulating Medium Wettability to Create Different Saturation Conditions
}

\author{
Ines Butz • Anna Herring
}

Received: date / Accepted: date

\begin{abstract}
Growing plants under microgravity conditions in a space ship is essential for future long term missions to supply needs for food and oxygen. Although plant growth modules for microgravity have been developed and tested for more than 40 years, creating optimal saturation conditions for plant growth in the absence of gravity still remains a challenge.

In this study, we present results from a series of spontaneous imbibition experiments designed to approximate microgravity conditions by using density matched fluid pairs. Porous media with patterned wettability characteristics are used to manipulate macroscopic fluid saturation and microscopic fluid interfacial configurations. These are compared to an additional experiment under Earth-gravity, wherein we observe spontaneous imbibition of water into common potting soil.

Patterning grains of different wettability under microgravity conditions proves to be an effective method to manipulate spatial distributions and saturations of fluids. These wettability patterns can be optimized to fine-tune residual fluid characteristics, e.g. non-wetting phase saturation, connectivity and interfacial area. Furthermore, we present tomographic evidence supporting previous work which was suggesting enhanced snap-off and disconnection of the gas phase in porous media under microgravity.
\end{abstract}

Keywords microgravity · wettability · spontaneous imbibition · porous media · multiphase $\cdot$ soil physics

Ines Butz

Department of Physics, Institute of Particle Physics and Astrophysics, Swiss Federal Institute of Technology, 8093 Zurich/

Department of Applied Mathematics, Research School of Physics and Engineering, Australian National University, Canberra, ACT 2601

E-mail: butz.ines@gmail.com

Anna Herring

Department of Applied Mathematics, Research School of Physics and Engineering, Australian National University, Canberra, ACT 2601

E-mail: anna.herring@anu.edu.au 


\section{Introduction}

Future long term space exploration missions will need a life support system as capacity for transporting resources is limited on a spaceship. Plants are proposed as an essential bioregenerative component in this system as they do not only produce food but also remove $\mathrm{CO}_{2}$ from the cabin atmosphere whilst providing $\mathrm{O}_{2}$ and treating waste water. The ability to provide optimal growth conditions for plants in non-terrestrial environments is not only important for future long term space missions but could also play a role for growing plants in reduced gravity environments e.g. on Mars.

During photosynthesis, plants use the sunlight to store energy in the form of adenosine triphosphate (ATP), taking up $\mathrm{CO}_{2}$ and water and releasing oxygen. To use this energy plants undergo respiration, where ATP is converted back to energy taking up oxygen and releasing $\mathrm{CO}_{2}$ and water (Hopkins (2006)). The processes of photosynthesis and respiration occur simultaneously in the green parts of a plant whereas the roots solely undergo respiration. As oxygen transport through the plant is slow, the necessary oxygen for respiration in the roots has to be taken up directly from the soil (Wegner (2010)).

In non-terrestrial environments conventional plant growth protocols used on Earth cannot be used as they result in insufficient supply of oxygen to the plants' roots: due to the lack of gravity fluid distributions in space drastically differ from those on Earth (Bingham et al (2000)). Plant growth systems have been developed and tested in space for more than 40 years, designed using different water and nutrient delivery systems. These range from completely passive systems, for instance bags or packs containing solidified agar (e.g Porterfield et al (1997), Massa et al (2017)), to active systems like perforated (e.g. Bingham et al (2000)) or porous tubes (e.g. Zhou et al (2002)) delivering nutrients directly to the plants' roots. Despite these efforts, reliably controlling water movement and distribution under microgravity still remains a challenge (Zabel et al (2016)).

One of the early growth modules tested in space was the SVET-Greenhouse 2 experiment, performed in 1996 (Bingham et al (1996)). That study demonstrated that the hydraulic conductivity in the experiment drastically differed to hydraulic conductivity on Earth which was explained by air bubbles getting trapped in the pore spaces of the growth media: on Earth, gravity dictates that pore spaces are filled from bottom to top by an invading fluid, whereas in the absence of gravity, the pore is filled by fluid moving along the sides of the pore due to capillary forces, which is illustrated in Figure 1. This exacerbates the entrapment of air, which impacts hydraulic conductivity as well as gaseous transport in the growth medium, leading to hypoxic conditions at the plants' roots (Bingham et al (2000)).

The most recent development is the VEGGIE growth unit installed on the International Space Station (ISS) in 2014 which was originally planned with a passive watering system. However, as this system fails to provide sufficient water, the plants have to be watered manually which is is not only leading to a higher crew involvement but also to suboptimal plant growth (Massa et al (2017)). The module has demonstrated the capability to grow plants in space as lettuce can be grown and eaten on the ISS. However, the challenges of growing plants in a microgravity fluid environment have been re-emphasized. Many multidisciplinary problems are to be solved, and one of the main question remains how to design 


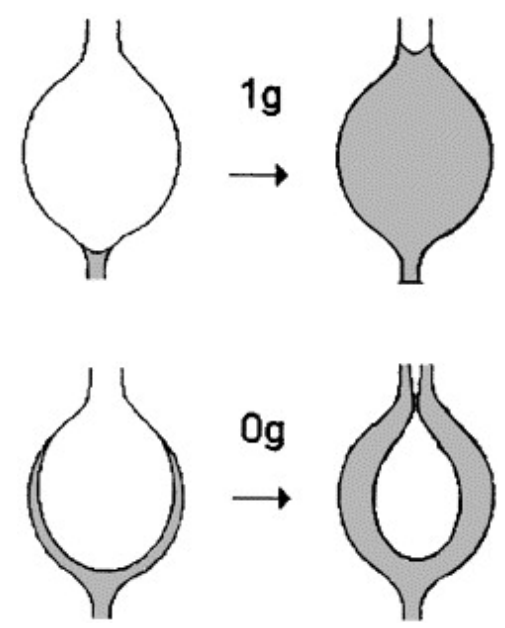

Fig. 1 Schematic of entrapment of air within a pore under microgravity. On Earth, an airfilled pore is filled from bottom to top whereas under microgravity a fluid can propagate along the walls of the pore, entrapping air. The Figure was taken from Bingham et al (2000).

a water and air management system for plant growth in space (Anderson and Quincy (2017)).

Apart from the experimental design of a growth module, fluid behaviour under microgravity has also been investigated theoretically. Chau and Or (2006) simulated drainage front morphology using the Lattice Boltzmann method for a range of gravitational forces. The corresponding gaseous diffusion coefficient was found to differ significantly between different flow regimes resulting from varying gravitational force. Jones and Or (1999) and Jones et al (2005) proposed an optimisation function to find optimal growth media parameters taking into account physiological requirements and physical system constraints. Dimensional analysis was used to find the optimal particle size in reduced gravity environments, e.g. lunar or Martian environments (Jones et al (2009)).

In this study, we consider an alternative method to provide optimal saturation conditions in microgravity conditions by controlling the wettability of the solid media substrate; we test the method by performing a series of spontaneous imbibition experiments in bead packs, where we define spontaneous imbibition as the process of fluid filling a solid matrix with no applied pressure driving the phase into the media. Many spontaneous imbibition experiments have been performed in the past in order to conduct research on fluid distributions in porous media on Earth, for example in the context of oil recovery research (Morrow and Mason (2001)); and while spontaneous imbibition is often examined in natural media like rock cores or sand packs, bead packs have been used as a model for an ideal porous medium (e.g. Meng et al (2016), Meng et al (2015), Li et al (2015), Hatiboglu and Babadagli (2008)). Gravity is known to have a significant influence the process of spontaneous imbibition in bead packs (Li and Horne (2001)) and larger unconsolidated granular materials like sand packs (e.g. as per analysis by Herring et al (2015)); for our microgravity-focused study we eliminate the effects of gravity 
through consideration of dimensional analysis.

Our method of controlling saturations and fluid distributions relies on manipulation of the porous medium wettability. Wettability is a fundamental factor influencing multiphase fluid flows in porous media (including air-water flows in soils), and is a measure of the preference of a solid to be in contact with one fluid rather than another (Abdallah et al (2007)). Considering a solid surface in contact with two fluids, a fluid with strong wettability to the surface will spread over it and displace the non-wetting fluid. The term fractional wettability refers to a porous medium containing surfaces with different wettability characteristics but does not imply specific locations of the differently wetting pores. Mixed wettability however refers to a special case of fractional wettability, which also implies a continuous pathway of one type of wettability throughout the medium (Salathiel (1973)). For the microgravity experiments presented here, the term fractional wettability applies to all packings, where some of the packings are also mixed-wet.

Packing beads of different wettability has been reported to influence the residual fluid distribution during forced imbibition (Celauro et al (2014)). Murison et al (2014) investigated fractionally wet bead packs with different correlation lengths between the same wettability type undergoing cycles of forced imbibition and drainage, and found that for high correlation lengths corresponding to clusters of same wettability beads, the front morphology becomes rather "rough". These previous studies indicate that spatial distribution of different wettability characteristics influences the fluid distribution. This study investigates how wettability patterning can be used to create different saturation conditions following spontaneous imbibition in porous media in the absence of gravity, applicable to designing a root growth medium for plants in space.

We have designed our experiments considering the suggestions of previous researchers in this area: first, as suggested by Or et al (2009), a porous plant growth environment should be designed with spatially segregated domains for water retention and gaseous flow; and second, a solid substrate solution is favourable in terms of reusability of scarce resources in space (Anderson and Quincy (2017)).

In greenhouse research it was found that a fraction of $10-15 \%$ of air contained in the soil is optimal for plant growth (Bunt (1988)). In soil, oxygen is primarily transported by diffusion, where diffusion within air is orders of magnitudes faster than within water. Numerical simulations (Armatas (2006))as well as theoretical considerations (Neira et al (2015)) suggest that the diffusion of oxygen through the air phase is strongly dependent not only on air-filled porosity but also on connectivity. Apart from connectivity, water-air interfacial area is also found to have an impact on oxygen transport in the soil. Gases are not only transported within air and water, but also exchanged between the phases, where the exchange rate scales linearly with air-water interfacial area (Skopp (1985)).

In our experiments, grains of different wettability characteristics are patterned within a 3D cylindrical column; and undergo spontaneous imbibition upon exposure to wetting fluid. Fluid dimensional analysis is used to approximate microgravity conditions in these experiments.

The fluid distributions obtained in both microgravity and Earth gravity experiments are characterised using state-of-the-art, high resolution, three dimensional X-ray microtomography (X-ray $\mu \mathrm{CT}$ ) at the CTLab at Australian National University. The $3 \mathrm{D}$ fluid distributions are quantified using geometrical and topological 
metrics with a focus on residual oil phase used in these experiments as a proxy for air. Number of residual clusters, spatial distribution as well as fluid interfacial area and connectivity are examined in order to characterise the effects of using different wettability in synthetic growth media.

\section{Methods}

\subsection{Materials}

\subsubsection{Fluids}

Microgravity conditions were created using fluid dimensional analysis via the Bond number Bo (Herring et al (2016)):

$$
B o=\frac{\text { gravity }}{\text { capillary }}=\frac{\Delta \rho \cdot g \cdot d^{2}}{\sigma \cdot \cos (\theta)}, 0^{\circ} \leq \theta<90^{\circ}
$$

where $\rho$ is the density, $g$ is the acceleration of gravity, $d$ is a characteristic length scale, $\sigma$ is the interfacial tension of the fluids, and $\theta$ is the fluid-fluid-solid contact angle. In space, the acceleration of gravity $g$ is equal to zero, resulting in $B o=0$. Therefore, the Bond number in the experimental system on Earth had to be close to zero in order to create microgravity conditions. This was achieved in our experimental system by choosing proxy fluids for water and air with a density difference $\Delta \rho$ close to zero.

Silicone oil and water were chosen as density-matched, immiscible fluid pair substituting for air and water under microgravity. Silicone oil with a viscosity of 100 cps was obtained (Wacker AK 100 Silicone Oil; Silchem International PTY Ltd.). Due to their similar density, X-ray attenuation of the fluids was very similar; so silicone oil was doped with the contrast agent iodoheptane at a concentration of $4 \%$ (volume).

To confirm the final densities of the fluid phases under experimental conditions, densities were measured manually: a volume of $0.5 \mathrm{ml}$ was measured using a $1 \mathrm{ml}$ syringe. The weight of the filled syringe was determined on a balance which had been calibrated using the weight of the empty syringe. This measurement was repeated 15 times for each fluid. From the average mass and the volume the density was computed. Using the measured fluid properties for doped silicone oil and water $\Delta \rho=0.011 \pm 0.029 \frac{\mathrm{g}}{\mathrm{cm}^{3}}, \sigma=56.16 \pm 0.42 \frac{\mathrm{mN}}{\mathrm{m}}$ and $\theta=14.1 \pm 1.3^{\circ}$ as contact angle between both fluids and protein coated glass, selecting the characteristic length scale to be the average bead diameter $a=0.655 \pm 0.055 \mathrm{~mm}$ and plugging in the accelaration of gravity on Earth $g=9.81 \frac{\mathrm{m}}{\mathrm{s}^{2}}$, Equation 1 yielded an experimental Bond number of $B o=0.00088 \pm 0.00015$. Errors were computed using Gaussian error propagation. The error of the mass measurement was determined by calculating the standard deviation of the measurements, whereas the error of the volume was assumed to be $0.01 \mathrm{ml}$ (smallest measuring unit of the syringe). Errors of interfacial tension $\sigma$ and contact angle $\theta$ were equal to the standard deviation in the respective measurements. The error of the characteristic length scale $a$ was chosen to be the difference between mean bead diameter and maximal/minimal 
bead diameter.

\subsubsection{Beads}

Bead packs were created using beads made from two different materials, where it was required that one of these materials is strongly wetting in order to induce spontaneous imbibition into the bead pack. Different materials were tested for their contact angle with respect to water in silicone oil in order to find strongly wetting materials using a goniometer. Protein coated surfaces were reported to be strongly wetting to water in silicone oil (Fawcett et al (1994)). A protein coating can be achieved using skim milk powder (Johnson et al (1984)). Glass which was immersed into a solution of $5 \%$ (mass concentration) skim milk powder in deionised water for 20 minutes was found to be strongly water-wet in the presence of silicone oil. A time series of contact angle measurements was taken, confirming the stability of the surface treatment over 48 hours and no inhomogeneities in wettabiliy were observed. PMMA was found to be neutrally wet with respect to silicone oil and water. Following the material tests, protein-coated soda lime glass and PMMA beads of $600-710 \mu \mathrm{m}$ diameter were selected for imbibition studies (Cospheric LLC, Santa Barbara, CA).

To enable re-use of the beads between experiments, glass and PMMA beads were separated and cleaned of silicone oil after each imbibition experiment. The oily, mixed beads were immersed in highly saturated brine with a droplet of dishwashing detergent. After stirring vigorously, PMMA beads floated on top of the solution and could be sieved out. Glass beads were cleaned with toluene, ethanol and water subsequently. As PMMA is not compatible with toluene, PMMA beads were cleaned several times using mineral turpentine. Contact angle measurements on a flat PMMA surface which had been cleaned using the same procedure were taken, verifying that the surface properties do not change due to cleaning procedures. Milk protein coating of the glass beads was repeated before every experiment.

\subsection{Experiments}

\subsubsection{Experimental Setup and Procedure}

A glass tube of $1.6 \mathrm{~cm}$ in diameter and $6.8 \mathrm{~cm}$ in length was filled with beads. The end caps were made using rubber stoppers with attached tubing. On the inside of the bottom rubber stopper a piece of water-wet paper (KimWipe) was attached to prevent the beads from falling into the tubing, but allowing water flow through it. The outlet of the upper rubber stopper was separated from the beads using a piece of oil-wet metal mesh which allowed oil flow, but prevented the PMMA beads from being flushed out. The water reservoir was always attached to the lower end of the flow cell. A valve was attached to the tubing at the bottom end connecting the tubing to a syringe. This syringe could be used to fill the tube with water, providing initial contact between water and protein coated glass beads. Note that a small section of beads at the base of the column (below the volume considered in the analysis) was initially saturated with water to ensure that the water supply 
at the inlet contacted the entirety of the bead pack diameter. The setup for a microgravity experiment is shown in Figure 2.

The bead pack was saturated with doped silicone oil by filling beads and fluid

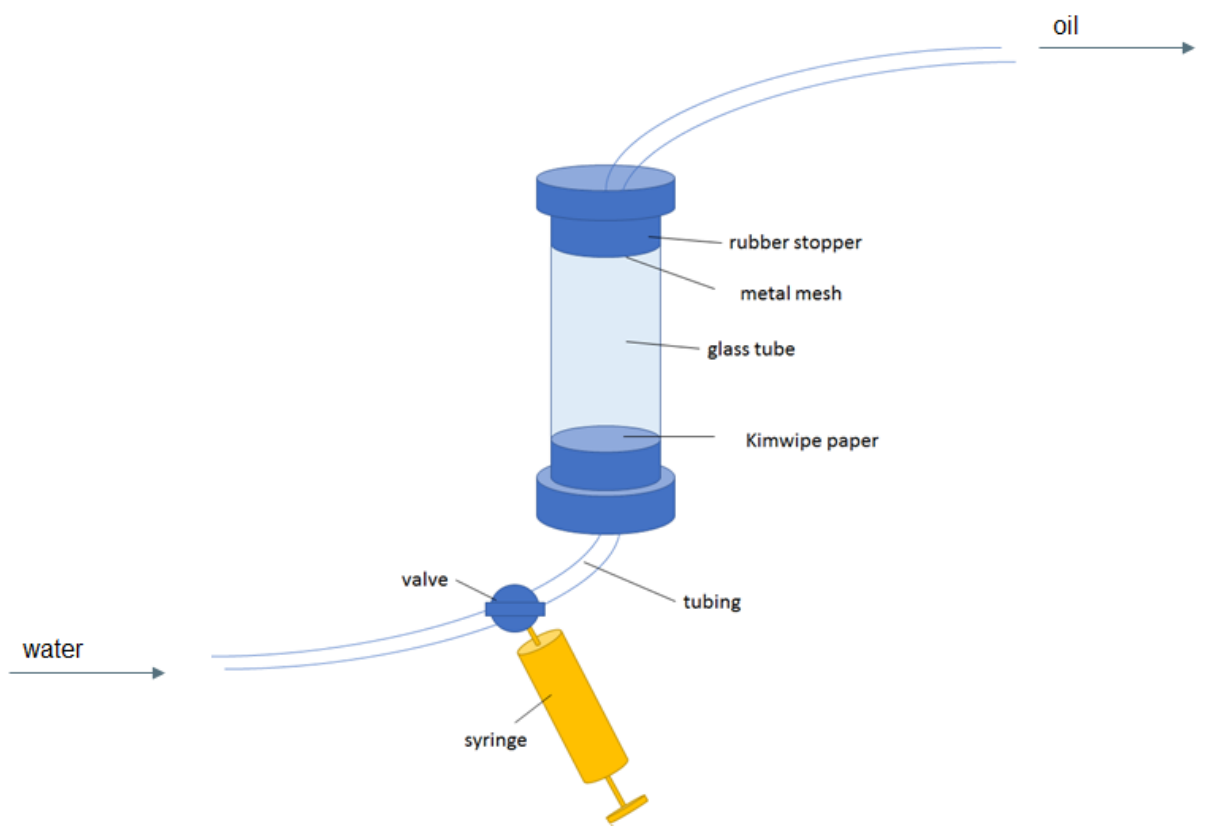

Fig. 2 Experimental setup: a glass tube filled with beads was sealed using rubber stoppers. To prevent the beads from being flushed into the tubing, Kimwipe paper and a metal mesh were used. Tubing was attached to connect the flow cell to the water and oil reservoir. A syringe was used to fill the tubing, providing initial contact between water reservoir and bead pack.

into the column simultaneously, paying carefully attention to avoid air bubbles in the packing. After the tubing was filled with the respective fluids providing hydraulic connection, the valve was opened to start the imbibition process. The reservoir beakers of silicone oil and water were positioned on adjustable stages, which were adjusted manually during the initial period of the imbibition, making sure both fluids are at the same level to avoid fluid flow due to hydrostatic pressure gradient. Both reservoirs were chosen to be shaped such that a small change in volume did not visibly decrease the fluid head; i.e. the pore volume of the bead column was much smaller than the fluid reservoirs. The fluids were given a time of $\geq 6$ hours to equilibrate before the valve was closed and the sample was imaged.

\subsubsection{Bead Packing}

For the microgravity experiments different patterns of glass and PMMA beads were packed, which can be seen in Figure 3. The first packing consisted of a linear distribution (approximately constant vertical gradient) of glass beads, starting from a concentration of $100 \%$ glass/ 0\% PMMA beads at the bottom, finishing 

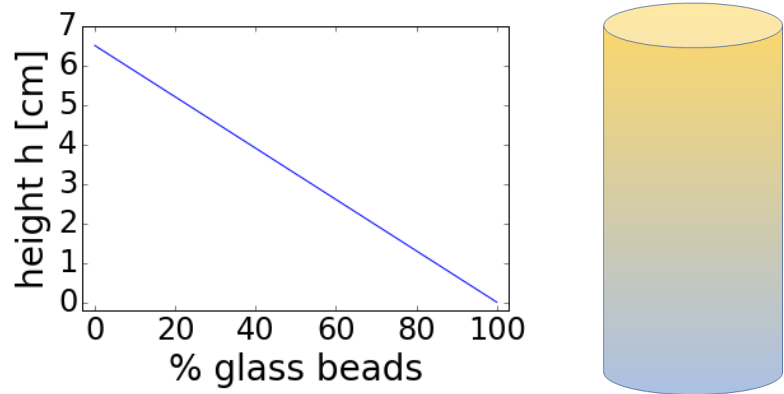

a) LinearRandom
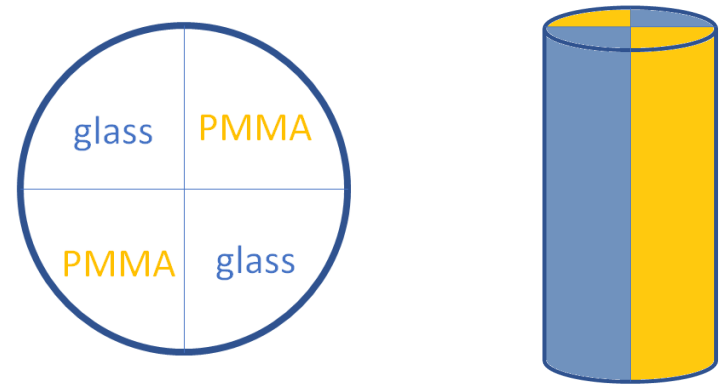

b) Quadrants
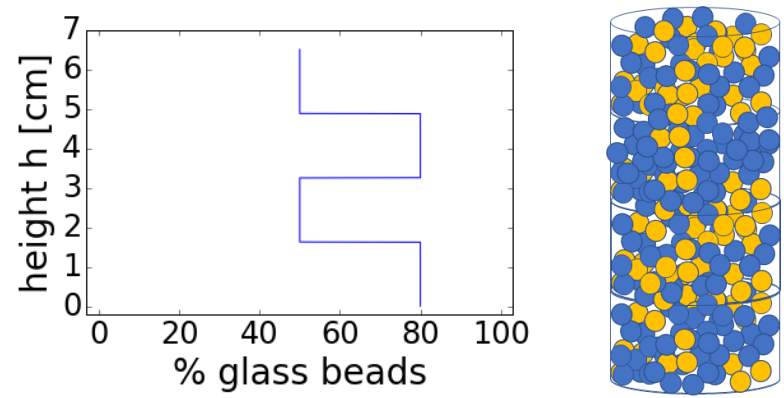

c) Layers

Fig. 3 Distribution of glass beads in the different samples. (a) fraction of glass beads decreases linearly from bottom to top; (b) cross section of the cylinder divided into quarters filled with $100 \%$ glass or PMMA beads respectively; (c) layers of 50\%/80\% of glass beads alternatingly.

at a concentration of $0 \%$ glass / $100 \%$ PMMA beads at the top (below referred to as "LinearRandom") (Figure 3 (a)). This packing was created by filling layers of $100 \%, 80 \%, 60 \%, 40 \%, 20 \%$ and $0 \%$ glass bead concentration into the cylinder subsequently. In each layer glass and PMMA was distributed randomly by stirring the mix of silicone oil, PMMA and glass beads before filling it into the cylinder. A second packing was prepared in which the cylinder was divided into quadrants, alternatingly filled with 100\% glass/PMMA (referred to below as "Quadrant") (Figure $3(\mathrm{~b})$ ). The quadrants were created using small partition walls, which were pulled out of the cylinder as it was filled. Due to density sorting effects of the 
beads, this packing did not result in perfect quadrants as shown in imaged data (included in the results, Section 3.2.2, Figure 8).

The final sample comprised a layered packing (below referred to as "Layers"). For this packing, equally sized vertical layers of $50 \%$ and $80 \%$ glass bead concentration were filled into the cylinder alternatingly (Figure 3 (c)).

\subsection{Reference experiment: Potting Soil under Earth gravity}

For the experiment under Earth gravity the same flow cell was used as for the microgravity experiments. Here, the cylinder was filled with common potting soil (initially saturated with air). The water reservoir was connected to the bottom end of the cylinder. The height of the water level was adjusted to be aligned with the water inlet to ensure that there was no water flow into the packing due to hydrostatic pressure.

The different packings and experimental conditions are listed in Table 1.

\begin{tabular}{l|l|l|l} 
packing name & imbibing fluid & $\begin{array}{l}\text { defending } \\
\text { fluid }\end{array}$ & materials \\
\hline $\begin{array}{l}\text { Earth-gravity } \\
\text { Potting Soil }\end{array}$ & water & air & common potting soil (ww) \\
\hline $\begin{array}{l}\text { microgravity } \\
\text { Linear RandomPack }\end{array}$ & water & silicone oil & $\begin{array}{l}\text { glass beads (protein coated) } \\
\text { (ww) }\end{array}$ \\
$\begin{array}{l}\text { Quadrants Milkbeads } \\
\text { Layers Milkbeads }\end{array}$ & water & silicone oil & $\begin{array}{l}\text { glass beads (protein coated) } \\
\text { (ww) }\end{array}$ \\
water & silicone oil & $\begin{array}{l}\text { Plass beads (protein coated) } \\
\text { (ww) }\end{array}$ \\
(wads beads (ow) \\
PMMA beads (ow)
\end{tabular}

Table 1 Overview of experimental conditions for all experiments. Water-wet/ oil-wet material is labelled (ww)/(ow).

\subsection{Imaging and Image Processing}

After the fluids had equilibrated for $\geq 6$ hours, the experiments were imaged via $\mathrm{X}$-ray $\mu \mathrm{CT}$. An aluminium filter was mounted in front of the detector to reduce beam-hardening.

Microgravity experiments were imaged at an X-ray energy of $100 \mathrm{keV}$ using an aluminium filter of $1.0 \mathrm{~mm}$ thickness. The potting soil experiment was imaged at an X-ray energy of $80 \mathrm{keV}$ using an aluminium filter of $0.5 \mathrm{~mm}$ thickness.

The reconstructed image was processed using the ANU-developed web-based software WebMango ${ }^{1}$. Smoothing and segmentation filters were applied in order to label all phases represented by different grayscale values in the tomogram. The processing work flow is explained in detail in Supplementary Materials S3.

\footnotetext{
1 https://webmango.anu.edu.au
} 


\subsection{Quantitative Analysis}

Phase profiles were recorded for each sample, measuring the fraction of each phase along the vertical axis of the sample. In microgravity experiments, due to low contrast between water and PMMA, these phases could not be digitally separated and were quantified as one phase. Similarly, in the Earth gravity experiment, the contrast between water and soil was very low. Thus, quantitative analysis was focused on the oil (air proxy) phase. Residual air/oil bubbles were quantified by measuring the number of clusters per volume, their number-volume distributions (see Supplementary materials) as well as their spatial distribution along the vertical axis of the sample. Additionally, the largest connected air/oil cluster was localised and visualised in a cross section of the sample.

In order to gain information not only about the macroscopic vertical distribution of fluids, but also about their microscopic characteristics, it is important to quantify the fluid's pore scale distribution. Herring et al (2015) use a topological invariant called Euler number $\chi$ to measure connectivity. A topological invariant is a quantity which is independent of continuous deformation (e.g stretching, bending, twisting), and so describes connectivity independent of geometry or size. The Euler number $\chi$ can be determined from the first three Betti numbers:

$$
\chi=\beta_{0}-\beta_{1}+\beta_{2},
$$

where $\beta_{0}$ describes the number of distinct fluid clusters, $\beta_{1}$ is the number of redundant connections within fluid clusters and $\beta_{2}$ is the number of holes within a fluid phase. For a highly connected and interconnected fluid the numbers of distinct fluid clusters is very small or one, whereas the number of redundant loops within the sample is very high. On the other hand, for a highly disconnected fluid there are many distinct fluid clusters with only few redundant connections. Therefore, a highly connected fluid corresponds to highly negative Euler number, whereas positive Euler numbers indicate low connectivity. The Euler number is a local quantity, determined for each cluster. Connectivity of the whole analysed volume is quantified by summing up all individual Euler numbers.

Another metric to measure connectivity is the Gamma function $\Gamma$, which in contrast to the Euler number, inherently weights clusters by their volume. The Gamma function is a global metric which is computed as follows:

$$
\Gamma(N W)=\frac{1}{n_{N W}^{2}} \sum_{i=1}^{N\left(X_{N W}\right)} n_{i}^{2}
$$

where $n_{N W}$ denotes the total number of non-wetting phase voxels in dataset $X_{N W}$, $N\left(X_{N W}\right)$ equals the total number of non-wetting phase clusters and $n_{i}$ is the number of voxels in non-wetting phase cluster $i$ (Renard and Allard (2013)).

Based on the traditional description of the terms "wetting" and "non-wetting phase", these terms do not apply in the microgravity experiments, as wettability varies throughout the sample. While strictly the oil is not a non-wetting phase, in this work the oil is referred to as non-wetting phase for clarity, referring to its non-wetting characteristics with respect to the glass beads. For all samples, connectivity metrics were applied to the air /oil (air proxy) phase and interfacial area was measured. As connectivity and interfacial area are known to be dependent on 
fluid saturation (e.g. Herring et al (2013), Culligan et al (2004)), the microgravity data sets were broken up into blocks of constant oil saturation and values were measured on each block individually.

\section{Results \& Discussion}

Spontaneous imbibition under standard Earth gravity conditions in potting soil is described in Section 3.1; this experiment provides context for the microgravity experiments, which are presented in Section 3.2. The acquired scans are presented and analysed with respect to relevant quantities for plant growth, e.g. distribution of the air (proxy) phase and connectivity.

Microgravity experiments were performed in order to investigate the possibility of manipulating fluid distribution using different wettability patterns. These oil (air proxy) fluid distributions measured under microgravity were compared to the the air distribution in potting soil under Earth gravity. Apart from analysing every sample qualitatively, the distribution of the air (proxy) phase was analysed quantitatively. The total number of air (proxy) clusters was counted in order to identify packings that facilitate entrapment. The spatial distribution of these entrapped air (proxy) clusters is quantified to verify influence of wettability on spatial fluid distribution respectively. Furthermore, connectivity and interfacial area of the residual air (proxy) clusters was quantified for microgravity experiments as these are considered to be parameters to optimise when engineering a synthetic plant growth medium.

\subsection{Potting Soil Experiment (Earth Gravity)}

\subsubsection{Acquired Scans and Qualitative Analysis}

Figure 4 shows a slice from the denoised tomogram, a slice from the processed data set in X-direction and a slice with air clusters colour labelled. The phase profile resulting from spontaneous imbibition of water in potting soil under Earth gravity quantifies the volumetric fraction of air and combined water and soil per slice in z-direction.

The scan of potting soil only allows for analysis of the air phase, as water and soil could not be distinguished due to similar attenuation. Air was present throughout the vertical extent of the potting soil, where the air fraction per slice exhibited variations of approximately $20 \%$ with a general trend of increasing air content towards the top of the cylinder (phase profile Figure 4 (d)). By labelling the air clusters it was found that the largest air cluster was percolating through the whole sample (pink cluster in Figure 4 (c)).

\subsubsection{Quantification of Fluid Distributions}

Distribution of Air Clusters Counting the isolated air clusters in the sample (refer to cluster volume histogram in Supplementary Materials) the total number of iso- 


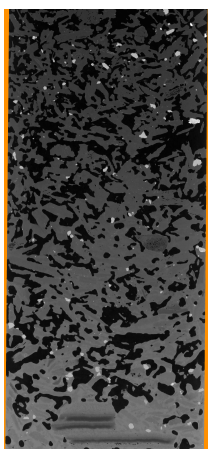

(a)

$\mathrm{X}$-slice (b) X-slice pro- (c) denoised tomo- cessed gram

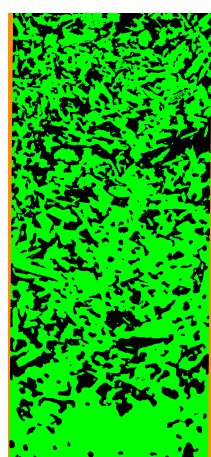

clusters

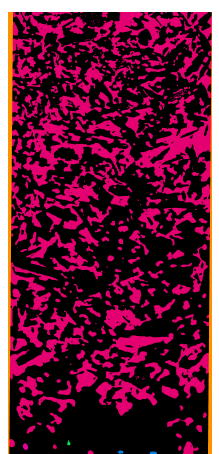

colour

air

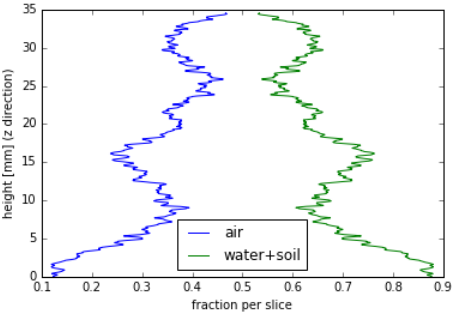

(d) phase profile

Fig. 4 Slice from the denoised tomogram (a) and the processed image (b) of the potting soil in X-direction, slice with isolated air clusters colour labelled (c) and phase profile of whole data set (d). Phases corresponding to the greyscales in tomogram/ colours in processed image are: air- black/black, Water+ potting soil-grey/green. It was not possible to distinguish water and soil in this scan. Edges of a mask applied to the data set are orange.

lated air clusters normalised by the imaged volume was found to be $0.0043 \frac{\text { clusters }}{\mathrm{mm}^{3}}$.

Figure 5 shows the position of the centre of gravity of air clusters in z-direction in the potting soil (y-axis, zero height is set to middle of the sample). The largest cluster in the sample was marked green, all other clusters are depicted in red. The size of the data set is indicated in the plot by horizontal lines which mark top and bottom of the scanned region. The size of each circle represents the volume of the respective cluster, where the radius of the circle is scaled logarithmically with the volume of the respective cluster.

Most air bubbles in the potting soil were located at the very bottom of the sample and only few isolated air bubbles were found around the reference height $z_{\text {mid }}$. As potting soil provides favourable conditions for growing plants on Earth, this distribution of isolated air bubbles should be approximately reproduced in an ideal synthetic growth medium under microgravity.

Connectivity and Interfacial Area Gas transport through the potential engineered growth media is another important feature to consider. In soil, gas transport is dominated by diffusion, which is not only dependent on the fraction of air-filled porosity but also on topological characteristics like connectivity or air-water interfacial area. These quantities were measured from the potting soil to establish a reference for engineered growth media under microgravity. At a mean air volumetric fraction of $33 \%$ (fraction of total volume including soil) connectivity and interfacial area are found to be:

- Connectivity measured by Euler number (normalised by total volume of the sample): $\chi=-5.9 \frac{1}{\mathrm{~mm}^{3}}$

- Connectivity measured by Gamma: $\Gamma=0.995$

- Interfacial area (normalised by total volume of the sample): $A=0.8 \frac{1}{\mathrm{~mm}}$ 


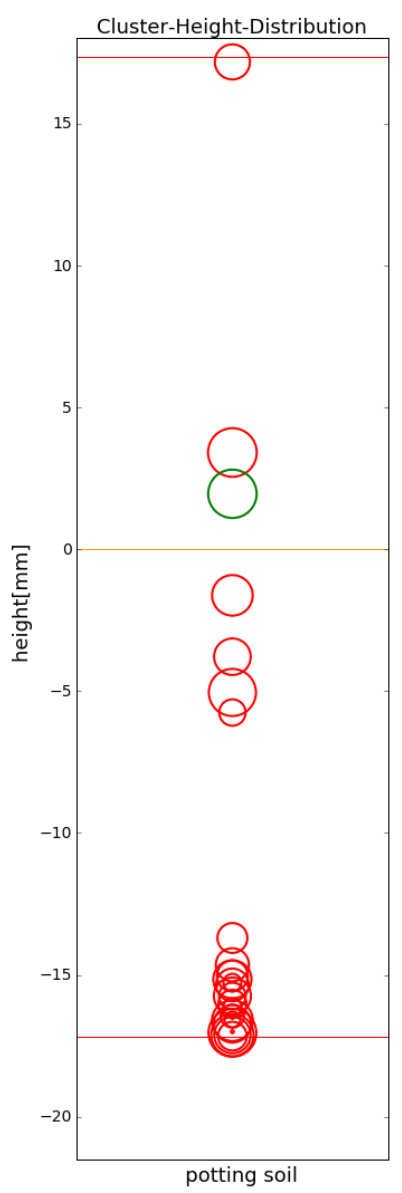

Fig. 5 Spatial distribution of isolated air clusters for the potting soil (red). The size of each circle represents the volume of the respective air cluster, where the radius of the circle is scaled logarithmically with the volume. The horizontal lines mark top and bottom of the scanned region.

\subsection{Microgravity Experiments}

\subsubsection{Microgravity Conditions}

As described in Section 2.1, the Bond number characterizing microgravity experiments was $\mathrm{Bo}=0.00088+/-0.00015$. Assuming an average grain size of $a=1 \mathrm{~mm}$ in soil growing roots and root hairs on Earth (Dexter (1988)), the Bond number for growing plants on Earth corresponds to:

$$
B o_{\text {Earth }}=0.136 \text {. }
$$

Here the literature values for the density of water $\rho_{\text {water }}=0.997 \frac{\mathrm{g}}{\mathrm{cm}^{3}}$, the density of air $\rho_{\text {air }}=0.001184 \frac{\mathrm{g}}{\mathrm{cm}^{3}}$, interfacial tension between air and water $\sigma=72 \frac{\mathrm{mN}}{\mathrm{m}}$ were used and a contact angle of $\theta=10^{\circ}$ was assumed for strongly water-wet 
material.

In space the acceleration of gravity $g$ equals zero resulting in:

$$
B o_{\text {space }}=0 .
$$

As displayed in Figure 6, the experimental Bond number was very close to zero, indicating that our experimental parameters were appropriate to simulate microgravity conditions. Note that the error determined for the experimental Bond number was too small to be visible in this plot.

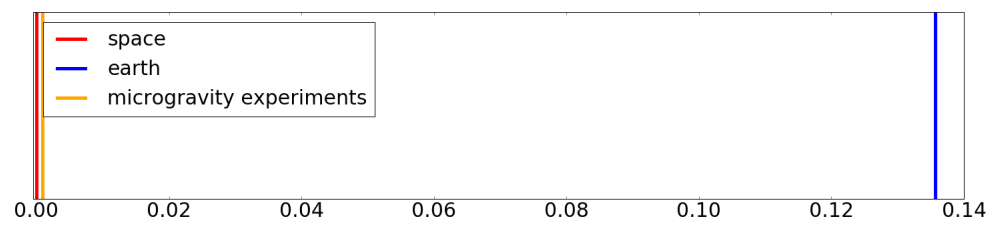

Fig. 6 Bond numbers for space, experiment and plant growth on Earth. The experimental Bond number was very close to zero compared to the Bond number on Earth, therefore the experiment is suitable for simulating microgravity conditions.

397

\subsubsection{Acquired Scans and Qualitative Analysis}

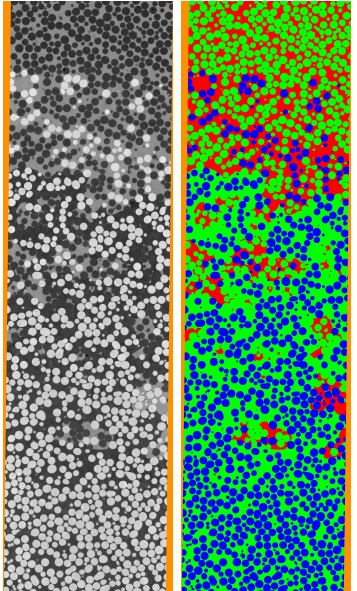

(a) X-slice (b) X-slice (c) tomogram

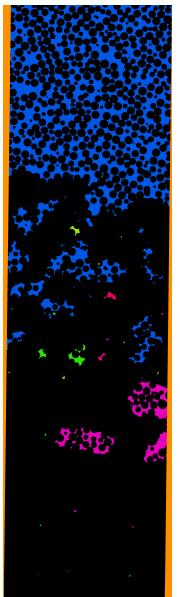

labelled oil clusters

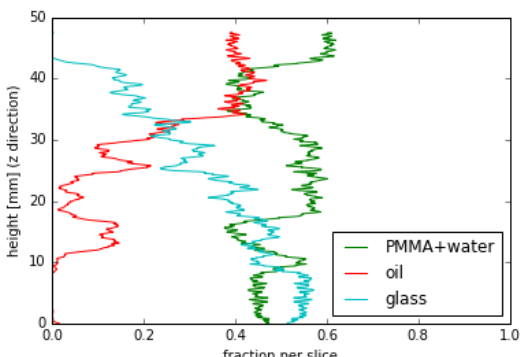

(d) phase profile

Fig. 7 Slice from the denoised tomogram (a) and the processed image (b) in X- direction, slice with isolated oil clusters colour labelled (c), phase profile of whole data set (d). Phases corresponding to the greyscales in tomogram/ colours in processed image are: dark/green: PMMA + water, grey/red: silicone oil, bright/blue: glass. Edges of a mask applied to the data set are orange. 
LinearRandom Packing The imbibition front is clearly visible in Figure 7, where water (as invading fluid) displaced the silicone oil; the fraction of silicone oil remaining in the system increases as glass bead fraction decreases. Although there were glass beads located directly above the front, the water did not invade further; this is potentially due to the fraction of water-wet glass beads decreasing below a certain critical fraction (we measured a ratio of $30.4 \%$ glass / 69.6\% PMMA beads in this experiment) necessary for water to imbibe. Residual oil bubbles are observed predominantly around PMMA beads: as PMMA beads are neutrally wet, they provide a relatively hospitable architecture to host silicone oil (in comparison to the water-wet glass beads).

Quadrant Packing A slice of the denoised tomogram and the segmented data is shown in Figure 8 (a) and (b) as well as a slice with colour labelled oil clusters (c) and a slice in z-direction (d). The phase profile of the whole sample is shown in (e).

The quarters, while slightly distorted, are clearly visible in Figure 8 (d): after imbibition, the glass bead section was saturated with water, whereas the PMMA section was filled with oil. In a perfect packing, the fraction of glass and PMMA beads across a slice in z-direction would be constant throughout the sample. However, the quarters were not perfectly shaped, with one of the PMMA quarters significantly growing in the top part of the sample; this was due to density sorting effects of the beads during packing. The water front stopped imbibing as the packing begins to become more PMMA-dominated, which suggests that a certain minimum width of the glass bead section is necessary for the front to progress. Residual oil bubbles are observed in the (strongly water-wet) glass bead section, this is likely due to the mechanism proposed by Bingham et al (2000) which is illustrated in Figure 1.

Horizontal Layers Packing Figure 9 shows that water imbibed to the top of the sample (in contrast to the LinearRandom packing described above) confirming that the critical glass bead fraction for water imbibition is below 50\%. A high amount of residual oil can be seen, which can again be explained by the presence of neutrally wet PMMA beads, which are relatively more hospitable to oil and tend to trap oil ganglia around them.

The phase profiles demonstrate that (surprisingly) the oil fraction was fairly constant throughout the whole Layers sample. Water imbibed to the top of the sample, leaving the same amount of residual oil behind independent of the glass bead fraction.

\subsubsection{Quantification of Fluid Distributions}

Distribution of Oil Clusters The measured number of isolated clusters for the microgravity experiments were: $0.10 \frac{\text { clusters }}{\mathrm{mm}^{3}}$ (LinearRandomPack), $0.11 \frac{\text { clusters }}{\mathrm{mm}^{3}}$ (QuadrantMilkbeads) and $0.45 \frac{\text { clusters }}{\mathrm{mm}^{3}}$ (Layers) (refer to cluster volume histogram in supplementary materials $\mathrm{S} 2$ ).

The total number of counted clusters per volume depended on the wettability 


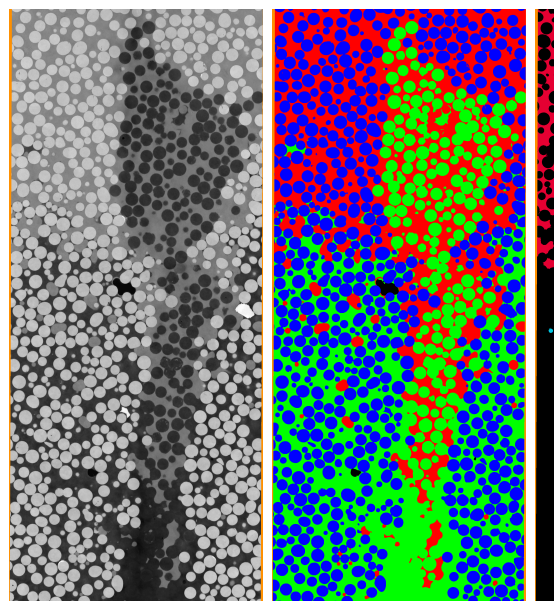

(a) X-slice de- (b) X-slice pro- (c) colour labelled noised tomogram cessed

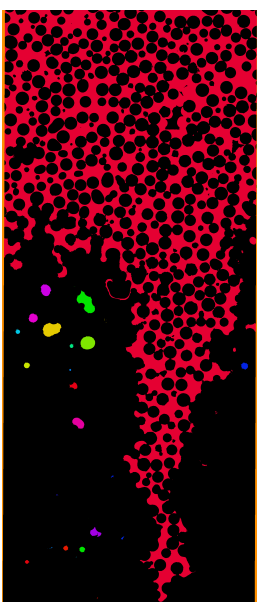
oil clusters

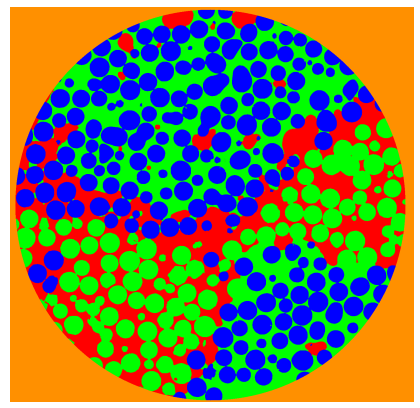

(d) Z-slice processed

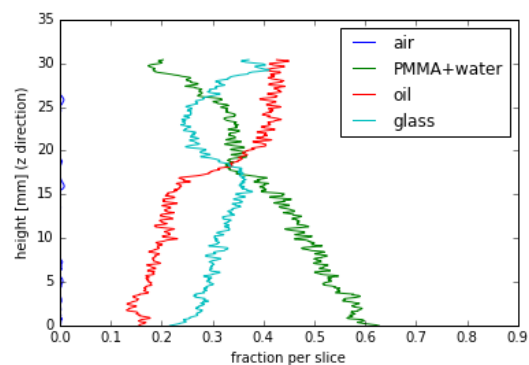

(e) phase profile

Fig. 8 Slice from the denoised tomogram in X-direction (a), processed image in X- and Zdirection (b) \& (d), slice with isolated oil clusters colour labelled (c) and phase profile of whole data set (e). Phases corresponding to the greyscales in tomogram/ colours in processed image are: black/black: air, dark/green: PMMA + water, grey/red: silicone oil, bright/blue: glass. Edges of a mask applied to the data set are orange.

pattern: a significantly higher number of isolated oil bubbles was found in the Layers experiment compared to both the LinearRandomPack and the Quadrant experiment. This difference was not due to image processing, as the same Isolated Cluster threshold on the oil phase is applied to all three data sets (for details on image processing refer to Supplementary Materials S3). Compared to the number of isolated air cluster measured in the potting soil under Earth gravity, the number of isolated air proxy clusters under microgravity is increased by a factor of 100 . This supports the idea of enhanced air entrapment due to increased layer flow (rather than piston-like displacement) under microgravity (Bingham et al (1996)).

The position of residual oil clusters was also measured. In the left-most column of Figure 10 each cluster is represented by a blue dot; its volume is represented on the $\mathrm{x}$-axis and its position (center of gravity) in z-direction is plotted on the 

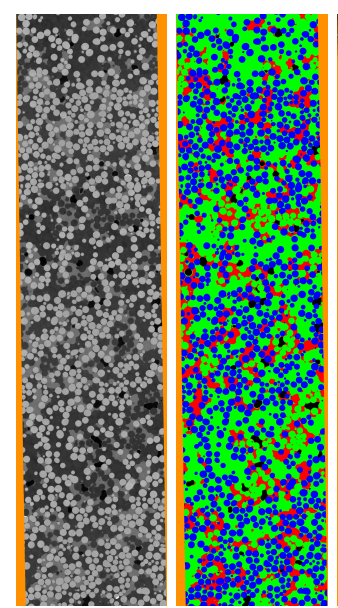

(a) X-slice denoised tomogram
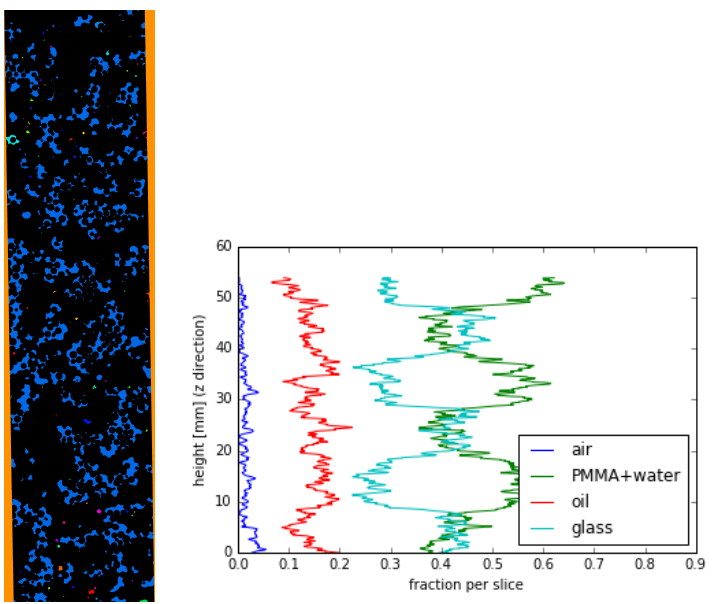

(d) phase profile

Fig. 9 Slice from the denoised tomogram and the processed image in $\mathrm{X}$ - direction, slice with isolated oil clusters colour labelled (c) and phase profile of whole data set (d). Phases corresponding to the greyscales in tomogram/ colours in processed image are: black/black: air, dark/green: PMMA + water, grey/red: silicone oil, bright/blue: glass. Edges of a mask applied to the data set are orange.

y-axis. As a high number of residual oil bubbles was measured, a histogram is displayed in the middle column; this displays the number of oil bubbles per interval in z-direction. In the right column, the corresponding phase profile is displayed, only showing the relevant phases oil and glass.

In Figure 10 the position of the largest cluster easily identified (i.e. it is the single cluster closest to the right edge of the left-most plot). Compared to the respective phase profiles (right-most plot), it is evident that for both the LinearRandomPack and the Quadrant experiment, the largest cluster was positioned at or slightly above the transition between glass and oil (intersection of blue and green line); this indicates that the oil cluster extended into the water filled section in the bottom of both samples. For the Layers experiment the location of the largest cluster was found at the vertical middle of the sample, indicating that in this sample the largest oil cluster percolated through the entire vertical extent of the sample. This observation is confirmed in the colour-labeled images presented above (Figures 7 (c), 8(c) and 9 (c)). The largest cluster can be easily identified to be: LinearRandom - blue, Quadrants - red, Layers - blue.

From the Layers experiment it is clear that the distribution of oil clusters is strongly correlated with glass bead concentration: the histogram peaks in sections of high glass bead concentrations, whilst the minima of the histogram are located in sections of low glass bead concentration. As the oil saturation was fairly constant throughout the sample, this indicates that in sections of high glass bead concentration many small oil clusters were left behind, whereas in sections of low glass bead concentration the same amount of oil was present in fewer but larger bubbles. For the Quadrants and the LinearRandomPack experiment there is no 

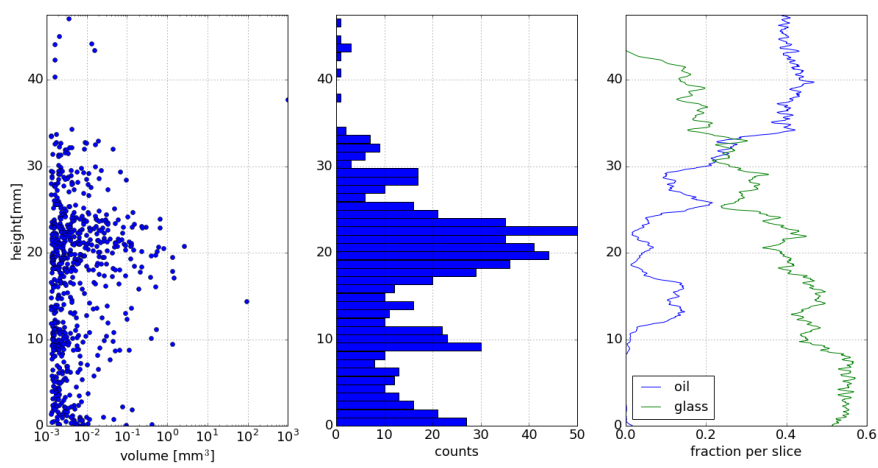

(a) LinearRandomPack
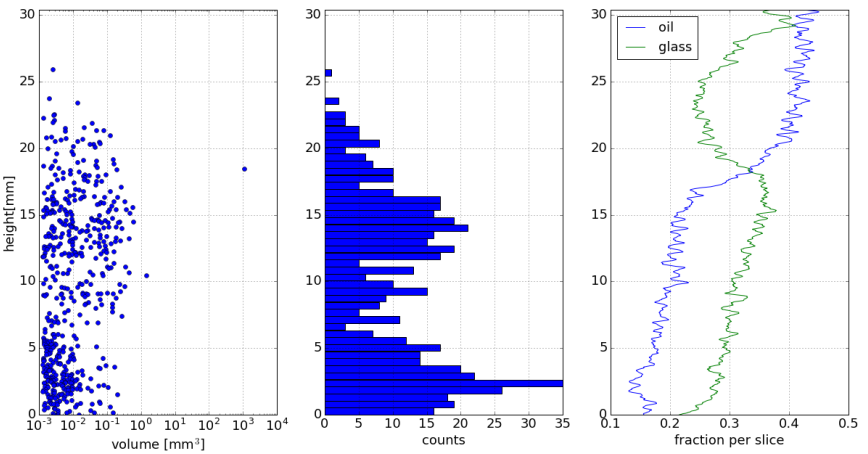

(b) Quadrants
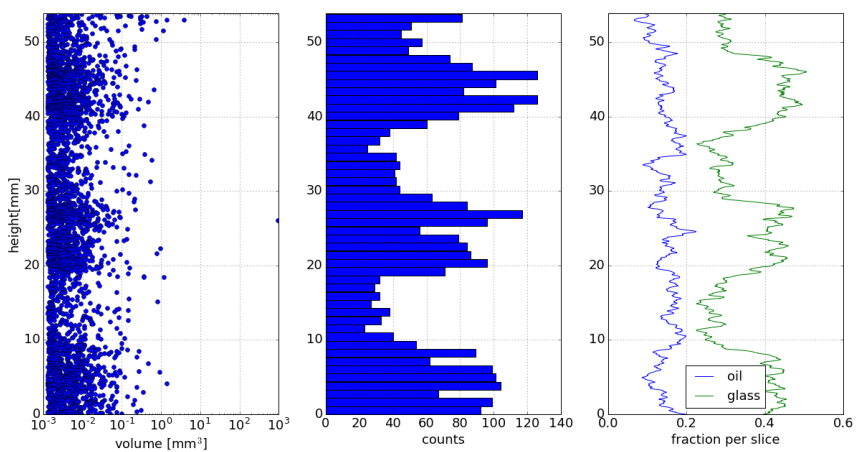

(c) Layers

Fig. 10 Volume and position of each isolated oil cluster (left), histogram counting the number of oil clusters per interval in z-direction (middle) and corresponding phase profile of oil and glass phases (right) in the microgravity experiments. 
obvious correlation between glass bead fraction and number of isolated bubbles. The number of clusters starts to increase slightly above the transition between oil and glass, peaking a few milimeters below this transition. At the bottom of the sample more variation in the cluster distribution is observed, which does not have an obvious dependency on oil and glass fraction present in this region. In these samples the distribution of oil clusters seems to be influenced by non-local effects: the glass bead configuration at a certain position influenced the number of residual oil bubbles, but so did the configurations above and below (e.g. many oil bubbles were present just below the transition of glass and oil fractions).

Connectivity and Interfacial Area For further analysis, the microgravity data sets were cut into saturation regimes. For the Quadrant and LinearRandomPack experiment these were sections on the phase profile where the fraction of oil only varied by a small amount. In the Layers experiment the fraction of oil only varied little over the whole sample. However, in order to examine whether the packing influenced the morphology of residual oil, the sample was broken up in blocks of similar glass bead density. The chosen regimes for each sample can be seen in Figure 11. The red line depicts the fraction of oil (fraction of glass beads for Layers) relative to the total volume per slice and the blue shaded regions are the chosen regimes. The mean fraction of oil was determined for each block. Figure 12 shows

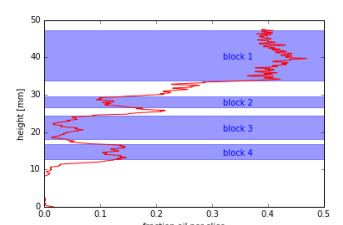

(a) LinearRandomPack

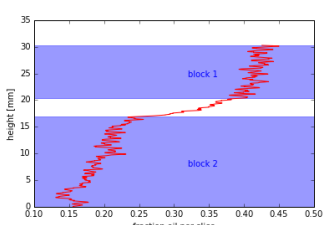

(b) Quadrants

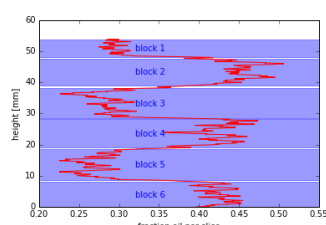

(c) Layers

Fig. 11 Chosen regimes of similar silicone oil saturation (fraction of glass beads for Layers) shaded in blue for all microgravity experiments.

the measurements performed on saturation blocks in microgravity experiments: Euler number (a) and Gamma function (b) were measured to quantify connectivity in the experiments. Euler numbers were normalised by volume of respective saturation regime and a reference point for maximal connectivity measured on a $100 \%$ saturated bead pack is provided (purple in Figure 12 (a)). Measured oil interfacial area is displayed in (c), again normalised by the volume of the respective saturation block. For all measurements each data point is labelled with the respective saturation regime where it was measured on. For reference, measured connectivity and interfacial area of the air phase in the Earth-gravity potting soil experiment are displayed (turquoise) with respect to the mean air-filled porosity in this sample.

For application to a plant growth medium, a highly negative Euler number (Equation 2) of the oil (gas proxy) phase is favourable; this indicates relatively few disconnected nonwetting bubbles (low $\beta_{0}$ ) and high nonwetting interconnectivity 


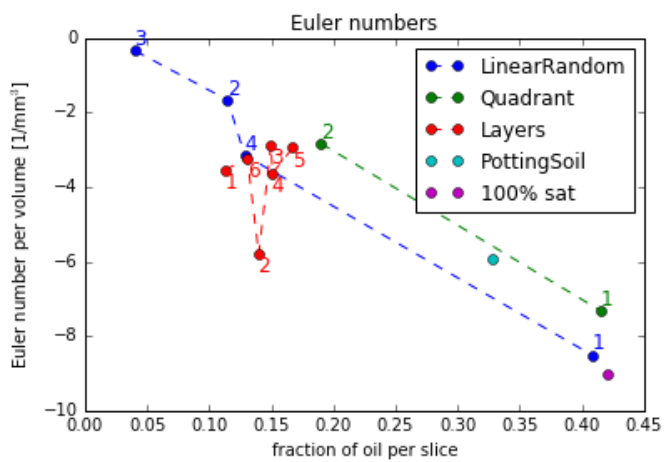

(a) Euler numbers

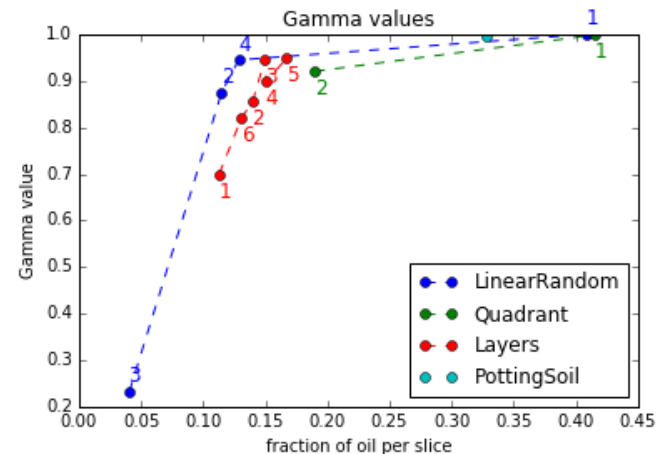

(b) Gamma function

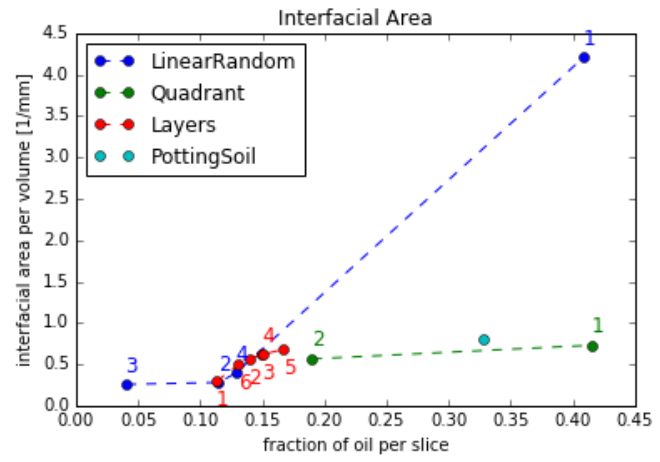

(c) Interfacial area

Fig. 12 Measured Euler number normalised by volume, Gamma function and interfacial area per volume for all saturation regimes in all three microgravity samples. Each data point is labelled by its corresponding saturation block. 
(high $\beta_{1}$ ). A high number of redundant loops $\beta_{1}$ within a fluid phase corresponds to a high number of pathways a fluid can take through the medium; and is therefore favourable for gas transport throughout the soil.

As shown in Figure 12 (a), the Quadrant packing consistently exhibits reduced interconnectivity (less negative $\chi$ ) relative to the LinearRandomPack. The oil phase in the LinearRandomPack almost reaches the maximal connectivity (purple data point in Figure 12 (a)), whereas the connectivity in the Quadrant packing is considerably reduced (less negative $\chi$ ), even at high saturation. The Layers experiment yields many data points around the same saturation. No clear trend is observable within this saturation regime, but the measured Euler numbers are in reasonable agreement with the connectivity measured on the LinearRandomPack. The connectivity of the air phase in potting soil under Earth gravity is slightly higher than in the Quadrants sample but reduced relative to the LinearRandomPack.

Looking at the connectivity values measured in the Layers packing is is interesting to observe that connectivity does not only depend on saturation and glass bead fraction, but also on the position of the blocks within the sample: Oil saturation was fairly constant throughout the sample and blocks $1,3,5$ as well as 2,4,6 are at same glass bead concentration. However, oil connectivity in these blocks decreases from top to bottom of the sample. We cannot currently confirm the mechanism responsible for this behaviour, but the observation indicates that there are nonlocal influences on fluid connectivity.

Figure 12 (b) also demonstrates that the LinearRandomPack provides the highest connectivity as measured by the gamma function (Equation 3). Two of the blocks within the Layers exhibit a similar connectivity as the LinearRandomPack. The other blocks, however, are significantly less connected with a difference $\Delta$ in $\Gamma(N W)$ of up to approximately $\Delta=0.2$ at the same oil saturation. The Quadrant experiment again exhibits a lower connectivity, however, the difference is less significant than for the Layers experiment (for the saturation ranges measured in this experiment). As measured by this connectivity metric, both the LinearRandomPack and the Quadrant experiment reach maximal connectivity for the highest saturation level achieved. Connectivity of the potting soil as measured by this metric is close to the maximal value (most of the air is connected in one big cluster). Both the LinearRandomPack and the Quadrants packing exhibit connectivities close to the potting soil at this saturation level.

In terms of plant growth, fluid interfacial area plays a role for gas transport across interfaces (gas transport by diffusion) between air and water, e.g. transport of $\mathrm{CO}_{2}$, where the exchange rate scales linearly with interfacial area between the phases (Skopp (1985)). Note, that as the water phase could not be isolated, the area measured in these experiments was the total oil interfacial area as opposed to the oil-water interfacial area. Figure 12 (c) demonstrates that there is a considerable difference between packings with randomly mixed glass and PMMA beads and packings with spatial separation between glass and PMMA. Both the LinearRandomPack and the Layers experiments contained randomly mixed glass and PMMA bead at various glass bead concentrations. For these packings the measurements of interfacial area of the oil phase coincide well. In the Quadrant experiment, which contained spatially separated PMMA and glass beads, the interfacial area is significantly smaller, especially at high oil saturation. This is supported by vi- 
sual observation of the images presented in Section 3.2.2: in samples with mixed PMMA and glass beads, oil was trapped around PMMA beads, creating complex oil clusters that winded around regions with high glass bead/water saturation. These tortuous geometries of clusters contribute to a high fluid interface. In the Quadrant experiment, however, oil and water mainly followed the spatial separation of glass and PMMA beads; therefore, oil and water were mainly present as large clusters in their respective quarters. This created rather flat oil interfaces as compared to the tortuous geometries in the mixed beads; thus resulting in a relatively lower interfacial area. However, interfacial area measured in the potting soil is close to the value obtained from the Quadrants packing. This suggests that interfacial area as exhibited in the Quadrants packing might be sufficient to ensure an appropriate level of gas exchange for plant growth.

\section{Conclusions}

An experimental setup was designed to investigate fluid distributions following spontaneous imbibition in porous media under microgravity conditions, which were designed considering dimensional analysis.

For reference, spontaneous imbibition of water in potting soil was examined under Earth-gravity conditions. Quantitative analysis focused on the number and position of entrapped air bubbles (isolated air bubbles have been identified as having negative effects on plant growth in previous studies). The potting soil was found to contain one large percolating air cluster and few isolated bubbles which were mostly located at the bottom of the sample.

In microgravity experiments, significantly more isolated non-wetting phase clusters were found, relative to the earth-gravity experiment. Therefore, these experiments provide tomographic evidence for observations made in early tests of growth modules in space suggesting that microgravity conditions enhance snapoff/disconnection of gas phase in porous media. The pattern of strongly water-wet glass beads was found to correlate with the spatial distribution of residual oil clusters. Furthermore, non-local effects were observed, suggesting that in addition to the immediately-local wettability pattern, the surrounding arrangement of fluids and solids had an impact on fluid entrapment.

By analysing the three different packings using topologic and geometric metrics, an impact of spatial wettability distribution on the measured characteristics was found. At the same non-wetting phase saturation a linearly decreasing fraction of water-wet material with height was found to provide the highest non-wetting phase connectivity as well as the highest interfacial area. At the same time, this packing exhibited the lowest density of small non-wetting phase clusters; this could be explained by the accompanying increasing fraction of non-water-wet material which provided a mobilization pathway for the non-wetting phase. A packing consisting of four quadrants of air-/water-wet material exhibited lower connectivity and interfacial area, however the measured values are still close to those measured in potting soil under Earth gravity for reference, suggesting that this packing might already be sufficient to provide appropriate conditions for plant growth. Based on these findings, future works could aim to design a plant growth medium which optimises all relevant characteristics of fluid distributions for plant growth. 
Note that these experiments model microgravity conditions, however the viscosity ratio of the chosen substituting fluids oil and water does not match the viscosity ratio of air and water. Generally, the system is dominated by capillary as opposed to viscous forces, as fluids were flowing at low velocities. Therefore, the trends observed in these experiments can be used to draw conclusions about fluid distributions under microgravity; however, an exact prediction of air-water microgravity distributions is not possible. The designed experimental setup provides a basis for future studies to develop an experiment approximating both microgravity conditions and the appropriate air-water viscosity ratio.

Overall, the microgravity experiments provide a proof of concept: manipulating fluid saturation is achievable in microgravity, by patterning grains of different wettability. The macroscopic spatial distribution of fluids can be manipulated as well as microscopic quantities important for plant growth (e.g. connectivity and interfacial areas).

Acknowledgements This study was enabled by the ANU Department of Applied Mathematics Stjepan Marcelja Visiting Fellowship, which provided financial support for experiments and travel for Ines Butz. Anna Herring is supported by the Australian Research Council with a Discovery Early Career Fellowship, DE180100082. We also wish to thank Jill Middleton, Michael Turner, Levi Beeching, Marie Jehannin, and Vince Craig for contributing to experimental design, data acquisition and processing.

Conflict of Interest: The authors declare that they have no conflict of interest.

\section{References}

Abdallah W, Buckley JS, Carnegie A, Edwards J, Herold B, Fordham E, Graue A, Habashy T, Seleznev N, Signer C, Hussain H, Montaron B, Ziauddin M (2007) Fundamentals of Wettability. Oilf Rev pp 44-61, DOI 10.6028/NBS.IR.78-1463, URL https://www.slb.com/ /media/Files/resources/oilfield_review/ors07/sum07/p44_61.pdf, arXiv:1011.1669v3

Anderson M, Quincy C (2017) Key Gaps for Enabling Plant Growth in Future Missions pp 1-18, DOI 10.2514/6.2017-5142

Armatas GS (2006) Determination of the effects of the pore size distribution and pore connectivity distribution on the pore tortuosity and diffusive transport in model porous networks. Chemical Engineering Science 61(14):4662 - 4675, DOI https://doi.org/10.1016/j.ces.2006.02.036, URL http://www.sciencedirect.com/science/article/pii/S0009250906001503

Bingham GE, Salisbury FB, Campbell WF, Carman JG, Bubenheim DL, Yendler B, Sytchev VN, Berkovitch YA, Levinskikh MA, Podolsky IG (1996) The Spacelab-Mir-1 Greenhouse-2 experiment. Adv Sp Res 18(4-5):225-232, DOI 10.1016/0273-1177(95)00881-E

Bingham GE, Jones SB, Or D, Podolski IG, Levinskikh MA, Sytchov VN, Ivanova T, Kostov P, Sapunova S, Dandolov I, Bubenheim DB, Jahns G (2000) Microgravity effects on water supply and substrate properties in porous matrix root support systems. Acta Astronaut 47(11):839-848, DOI 10.1016/S00945765(00)00116-8 
Bunt AC (1988) Media and mixes for container-grown plants. (A manual on the preparation and use of growing media for pot plants). Ed. 2, Unwin Hyman, London, UK

Celauro JG, Torrealba VA, Karpyn ZT, Klise KA, Mckenna SA (2014) Pore-scale multiphase flow experiments in bead packs of variable wettability. Geofluids 14(1):95-105, DOI 10.1111/gfl.12045

Chau JF, Or D (2006) Linking drainage front morphology with gaseous diffusion in unsaturated porous media: A lattice Boltzmann study. Phys Rev E - Stat Nonlinear, Soft Matter Phys 74(5), DOI 10.1103/PhysRevE.74.056304

Culligan KA, Wildenschild D, Christensen BSB, Gray WG, Rivers ML, Tompson AFB (2004) Interfacial area measurements for unsaturated flow through a porous medium. Water Resources Research 40(12), DOI 10.1029/2004WR003278, URL https://agupubs.onlinelibrary.wiley.com/doi/abs/10.1029/2004WR003278, https://agupubs.onlinelibrary.wiley.com/doi/pdf/10.1029/2004WR003278

Dexter A (1988) Advances in characterization of soil structure. Soil Tillage Res 11(3-4):199-238, DOI 10.1016/0167-1987(88)90002-5

Fawcett IM, Williams RL, Wong D (1994) Contact angles of substances used for internal tamponade in retinal detachment surgery. Graefe's Arch Clin Exp Ophthalmol 232(7):438-444, DOI 10.1007/BF00186587

Hatiboglu CU, Babadagli T (2008) Pore-scale studies of spontaneous imbibition into oil-saturated porous media. Phys Rev E - Stat Nonlinear, Soft Matter Phys 77(6), DOI 10.1103/PhysRevE.77.066311

Herring AL, Harper EJ, Andersson L, Sheppard A, Bay BK, Wildenschild D (2013) Effect of fluid topology on residual nonwetting phase trapping: Implications for geologic co2 sequestration. Advances in Water Resources 62:47 - 58, DOI https://doi.org/10.1016/j.advwatres.2013.09.015, URL http://www.sciencedirect.com/science/article/pii/S0309170813001760

Herring AL, Andersson L, Schlüter S, Sheppard A, Wildenschild D (2015) Efficiently engineering pore-scale processes: The role of force dominance and topology during nonwetting phase trapping in porous media. Adv Water Resour 79:91-102, DOI 10.1016/j.advwatres.2015.02.005

Herring AL, Sheppard A, Andersson L, Wildenschild D (2016) Impact of wettability alteration on $3 \mathrm{~d}$ nonwetting phase trapping and transport. International Journal of Greenhouse Gas Control 46:175 - 186, DOI https://doi.org/10.1016/j.ijggc.2015.12.026

Hopkins W (2006) Photosynthesis and Respiration. The Green World, Facts On File, Incorporated

Johnson DA, Gautsch JW, Sportsman JR, Elder JH (1984) Improved technique utilizing nonfat dry milk for analysis of proteins and nucleic acids transferred to nitrocellulose. Gene Anal Tech 1(1):3-8, DOI 10.1016/0735-0651(84)90049-9

Jones SB, Or D (1999) Particulated growth media for optimal liquid and gaseous fluxes to plant roots in microgravity. In: Instrum. Monit. Control Celss, vol 22, pp 1413-1418, DOI 10.1016/S0273-1177(98)00221-X

Jones SB, Heinse R, Bingham GE, Or D (2005) Modeling and Design of Optimal Growth Media from Plant-Based Gas and Liquid Fluxes. SAE Tech Pap 200501-29, DOI 10.4271/2005-01-2949

Jones SB, Bugbee B, Heinse R, Bingham GE (2009) Porous Plant Growth Media Design Considerations for Lunar and Martian Habitats. SAE Int Pap 01- 
2361(1):8pp, DOI 10.4271/2009-01-2361

Li G, Chen X, Huang Y (2015) Contact Angle Determined by Spontaneous Imbibition in Porous Media: Experiment and Theory. J Dispers Sci Technol 36(6):772777, DOI 10.1080/01932691.2014.921627

Li K, Horne RN (2001) Characterization of Spontaneous Water Imbibition Into Gas-Saturated Rocks. SPE J 6(04):375-384, DOI 10.2118/74703-PA, URL http://www.onepetro.org/doi/10.2118/74703-PA

Massa GD, Newsham G, Hummerick ME, Morrow RC, Wheeler RM (2017) Plant Pillow Preparation for the Veggie Plant Growth System on the International Space Station. Gravitational Sp Res 5(1), URL http://gravitationalandspacebiology.org/index.php/journal/article/view/749

Meng Q, Liu H, Wang J (2015) Entrapment of the non-wetting phase during Co-current spontaneous imbibition. Energy and Fuels 29(2):686-694, DOI 10.1021/ef5025164

Meng Q, Liu H, Wang J (2016) Effect of Viscosity on Oil Production by Cocurrent and Countercurrent Imbibition From Cores With Two Ends Open. SPE Reserv Eval Eng (May), DOI 10.2118/183635-PA, URL http://www.onepetro.org/doi/10.2118/183635-PA

Morrow NR, Mason G (2001) Recovery of oil by spontaneous imbibition. DOI 10.1016/S1359-0294(01)00100-5

Murison J, Semin B, Baret JC, Herminghaus S, Schröter M, Brinkmann M (2014) Wetting Heterogeneities in Porous Media Control Flow Dissipation. Phys Rev Appl 2(3), DOI 10.1103/PhysRevApplied.2.034002, arXiv:1310.3125v2

Neira J, Ortiz M, Morales-Salinas L, Acevedo E (2015) Oxygen diffusion in soils: Understanding the factors and processes needed for modeling. Chilean journal of agricultural research 75:35-44, DOI 10.4067/S0718-58392015000500005

Or D, Tuller M, Jones SB (2009) Liquid Behavior in Partially Saturated Porous Media under Variable Gravity. Soil Sci Soc Am J 73(2):341, DOI 10.2136/sssaj2008.0046, URL https://www.soils.org/publications/sssaj/abstracts/73/2/341

Porterfield DM, Matthews SW, Daugherty CJ, Musgrave ME (1997) Spaceflight exposure effects on transcription, activity, and localization of alcohol dehydrogenase in the roots of Arabidopsis thaliana. Plant Physiol 113(95):685-693, DOI 10.1104/pp.113.3.685

Renard P, Allard D (2013) Connectivity metrics for subsurface flow and transport. Adv Water Resour 51:168-196, DOI 10.1016/j.advwatres.2011.12.001

Salathiel R (1973) Oil Recovery by Surface Film Drainage In MixedWettability Rocks. J Pet Technol 25(10):1216-1224, DOI 10.2118/4104-PA, URL http://www.onepetro.org/doi/10.2118/4104-PA

Skopp J (1985) Oxygen uptake and transport in soils: Analysis of the air-water interfacial area1. Soil Science Society of America Journal - SSSAJ 49, DOI 10.2136/sssaj1985.03615995004900060001x

Wegner LH (2010) Oxygen transport in waterlogged plants. In: Waterlogging Signal. Toler. Plants, pp 3-22, DOI 10.1007/978-3-642-10305-6_1

Zabel P, Bamsey M, Schubert D, Tajmar M (2016) Review and analysis of over 40 years of space plant growth systems. DOI 10.1016/j.lssr.2016.06.004

Zhou W, Durst SJ, Demars M, Stankovic B, Link BM, Tellez G, Meyers RA, Sandstrom PW, Abba JR (2002) Performance of the Advanced A STROCULTURE Plant Growth Unit During ISS-6A / 7A Mission. 32nd Int Conf Environ Syst 


\section{Supplementary Materials}

\section{S1 Contact angle measurements}

In Figure S1 a droplet of water in silicone oil is shown on a untreated PMMA surface as well as on a protein coated glass surface. From these measurements it can be concluded that the treated glass is strongly water-wet in silicone oil (contact angle $\theta_{\text {glass }}=20$ ) whereas the PMMA is neutrally wet (contact angle $\theta_{\mathrm{PMMA}}=$ $111^{\circ}$ ). For the protein coated glass a time series of contact angle measurements was taken to ensure that the contact angle does not considerably change during the experiment. Contact angle measurements of the coated glass yielded consistent contact angles implying that the coating was sufficiently uniform.

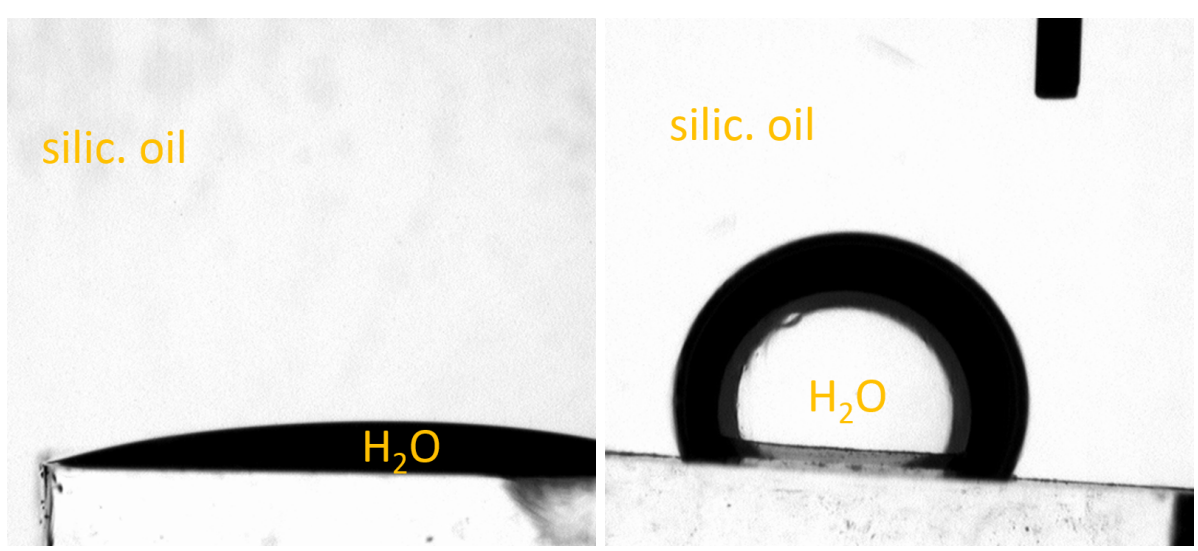

(a) protein coated glass (b) untreated PMMA

Fig. S1 Contact angle measurements of water on untreated glass and PMMA in silicone oil.

\section{S2 Cluster Volume histograms}

\section{S2.1 Earth-gravity}

In Figure S2 the measured cluster volume histogram is shown for the potting soil. It is important to note that due to the Isolated Cluster filter which was used to remove noise in the segmented data, no clusters could be counted below the threshold set for this filter. This threshold was set to 10000 voxels, corresponding to a volume of approximately $0.005 \mathrm{~mm}^{3}$. The threshold was chosen such that all noise was removed while preserving real features.

The histogram in Figure S2 demonstrates that most of the isolated clusters were found between a volume of about 0.1 and $1.0 \mathrm{~mm}$. Furthermore there was one air cluster that was orders of magnitude larger than the other clusters. 


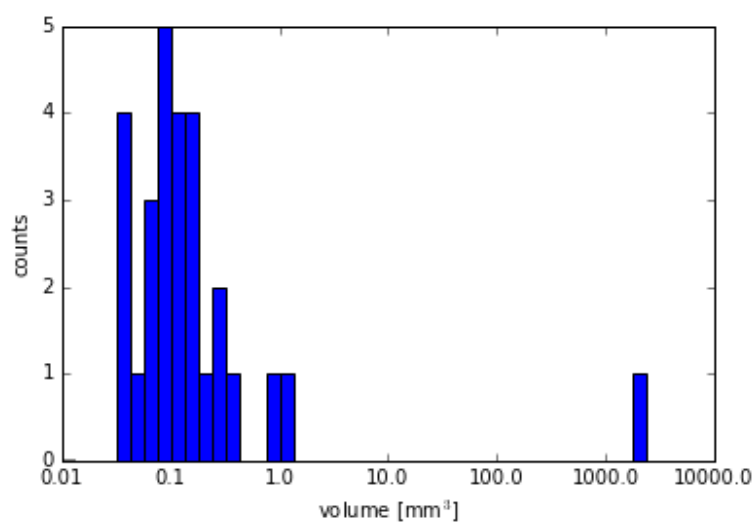

Fig. S2 Number of clusters counted within each volume interval for the potting soil experiment.

\section{S2.2 Microgravity}

The volume of each individual oil cluster was measured for all microgravity samples, shown as cluster size histograms in Figure S3. For microgravity experiments, many very small residual oil bubbles were present in the samples, some of which were unfortunately digitally removed during necessary post-segmentation noise removal. To ensure consistency between all samples, the removal size thresholds on the oil phase were kept constant for all samples; the threshold value was chosen to be the smallest number that removed most of the noise whilst still preserving real small oil bubbles (in these experiments, a volume of 2000 voxels was used, which corresponds to approximately $0.001 \mathrm{~mm}^{3}$ ). For this reason, some of the clusters which would be counted in the first volume bin of the cluster size histograms (Figure S3) were removed, resulting in smaller counts for this interval. As shown in

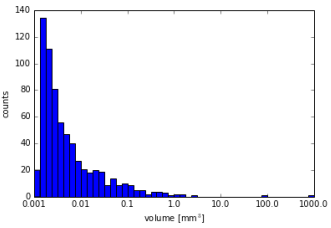

(a) LinearRandomPack

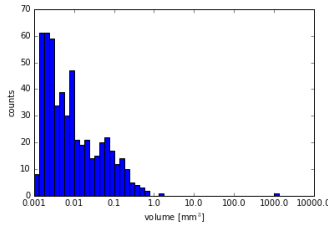

(b) Quadrants

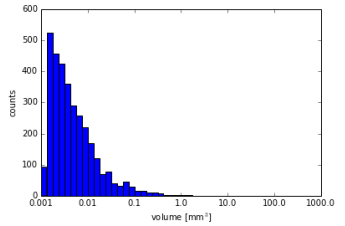

(c) Layers

Fig. S3 Number of clusters counted within each volume interval for all three microgravity experiments.

the cluster volume histograms, most residual oil clusters had a volume between 0.001 and $0.01 \mathrm{~mm}^{3}$; this is significantly smaller than the air bubbles found in the Earth-gravity experiments. 
S3 Image Processing

\section{S4 Image Processing Parameters}

Tables S1 to S4 show parameters chosen for image processing steps, where parameters had to be varied between data sets. Table S2 shows the chosen segmentation

\begin{tabular}{l|l|l} 
sample & radius $(\mathrm{GM})$ [voxels] & contrast $(\mathrm{AD})$ \\
\hline PottingSoil & 895 & 1500 \\
\hline Linear RandomPack2 & 750 & 300 \\
\hline Quadrants Milkbeads & 746 & 2000 \\
\hline Layers Milkbeads & 746 & 300
\end{tabular}

Table S1 Radii of the smallest Geometric Mask applied to the data set and contrast threshold chosen for the Anisotropic Diffusion filter.

The processing work flow is shown in Figure S4. Note that the data set presented as example in this Figure was not included in the final analysis, however the same box containing all non-masked voxels was founc and Anisotropic Diffusion filters were used to remove noise and enhance edges. As a next step the data was segmented using thresholds determined from the intensity histogram of the tomograms (see Supplementary Materials S4). The filter Isolated Clusters was used to remove small clusters of mislabeled voxels (Figure S4 c), yellow arrows). For the potting soil experiment, fluid-fluid interfaces were ched; however, for microgravity experiments no additional smoothing was applied to avoid removal of narrow features in these data sets. All chosen thresholds and parameters for processing steps are detailed in the next section.

thresholds for both Earth and microgravity scans. Main thresholds were chosen using the peak-to-minimum relations explained below and displayed in Figure S5. Gradient thresholds were chosen for each data set individually. In order to ensure 


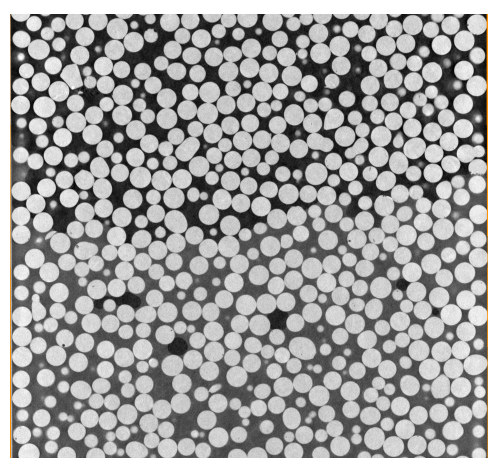

(a) Geometric Mask \& AutoCrop

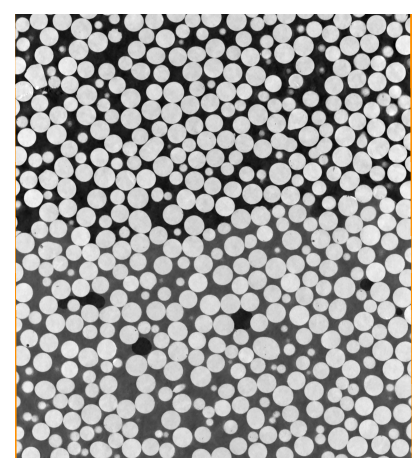

(b) Bilateral Iteration \&

Anisotropic Diffusion

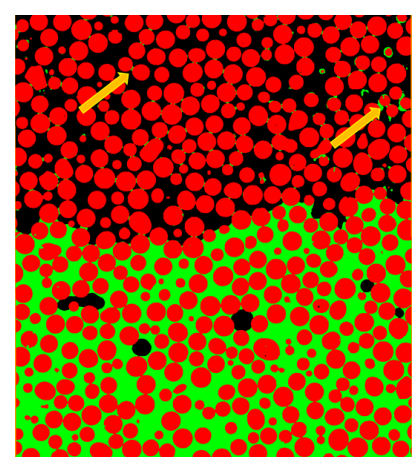

(c) Segmentation

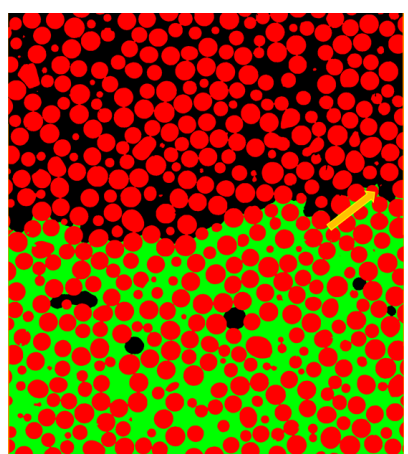

(d) Isolated Clusters

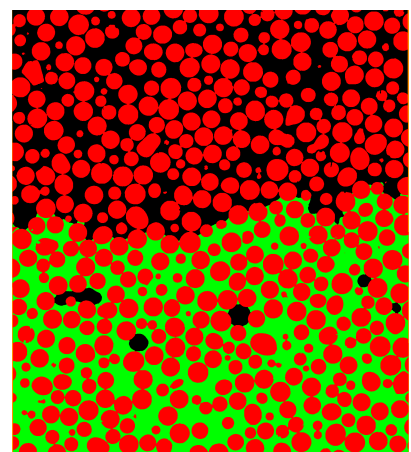

(e) Smooth Segmented Data

Fig. S4 Slice through a bead column after each processing step (data set not presented in this work, however, the same processing steps were performed on all presented data sets). Note that an additional Geometric Mask was applied after (a) cropping off two bead diameters from the walls in order to be able to ignore wall effects, resulting in narrower images (b)-(d). Yellow arrows point out examples of artefacts that are removed in the following processing step. 


\begin{tabular}{l|l|l}
\hline sample & main treshold & gradient thresholds \\
\hline PottingSoil & Earth thresholds (for seg1) & none \\
\hline LinearRandomPack & microgravity thresholds & $\begin{array}{l}\text { seg1: 20 on glass } \\
\text { seg2: 20 on oil }\end{array}$ \\
\hline Quadrants & $\begin{array}{l}\text { microgravity thresholds } \\
\text { seg1 and seg2) } \\
\text { to artifacts }\end{array}$ & $\begin{array}{l}\text { seg1: 20 on glass } \\
\text { seg2: 20 on oil } \\
\text { seg3: 200 on PMMA+water }\end{array}$ \\
\hline Layers & microgravity thresholds & $\begin{array}{l}\text { seg1: 20 on glass } \\
\text { seg2: 20 on oil } \\
\text { seg3: 50 on PMMA+water }\end{array}$
\end{tabular}

Table S2 Segmentation thresholds chosen during image processing of earth-gravity and microgravity data sets.

consistency between different datasets, thresholds for the Earth-gravity data sets were chosen as follows: on the bead pack dataset (preliminary experiment, not included in this work), thresholds were chosen by eye and tested. The thresholds determined in this process were found to preserve narrow features like fluid in pore throats. At the same time they did not produce artifacts like rings around beads due to partial volume effects. After having achieved a satisfying segmentation, the position of the chosen thresholds on the intensity histogram of the image were determined (see Figure S5 a)): the thresholds (turquoise) for a segmentation are always positioned between two intensity peaks (red), which are separated by an intensity minimum (green). The distances between both peaks and the minimum was determined (arrows labelled $d_{1}$ to $d_{4}$, orange: segmentation 1, blue: segmentation 2). Then the distances between maxima and thresholds was determined as well as its percentage of the total maximum-minimum distance (arrows labelled with percentages). In all subsequent segmentations of datasets from Earth gravity experiments (only potting soil presented here), these same percentages were used in order to determine the positions of thresholds relative to intensity extrema consistently.

Gradient thresholds could not be determined universally for all datasets and were chosen manually.

For microgravity experiments, the same procedure was used to chose segmentation thresholds consistently. These data sets contained five phases: air, water, PMMA, doped silicone oil and glass. The attenuation of water and PMMA was too similar for these phases to be segmented, therefore they were taken together as one phase. The amount of air in these data sets was very small, as the entrapment of air during packing was avoided as much as possible, therefore the corresponding peak was very small (or not existent at all for some data sets). Figure S5 b) demonstrates the peak - minimum relations used to determine segmentation thresholds for microgravity experiments. For the Quadrant packing, a separate threshold had to be chosen for the air phase due to imaging artifacts caused by strong beam hardening in the glass sections of the packing. Gradient thresholds were again determined manually for each data set.

Table S3 shows the thresholds for the Isolated Clusters filter for the Earth-gravity data set, Table S4 for microgravity data sets. 


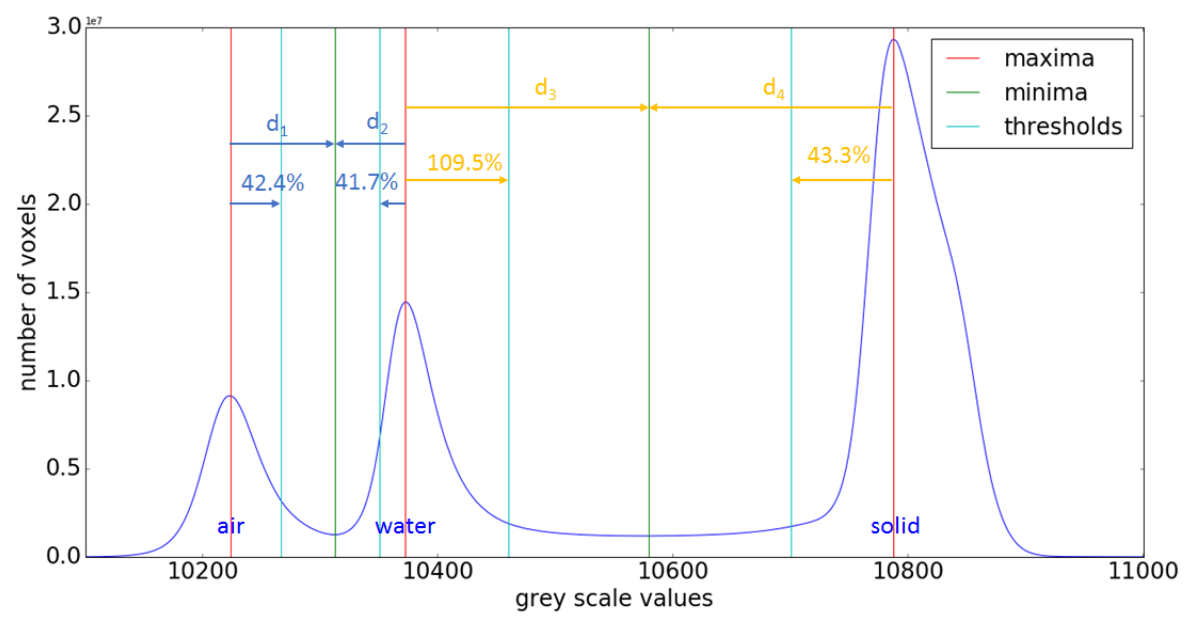

(a) Earth-gravity thresholds

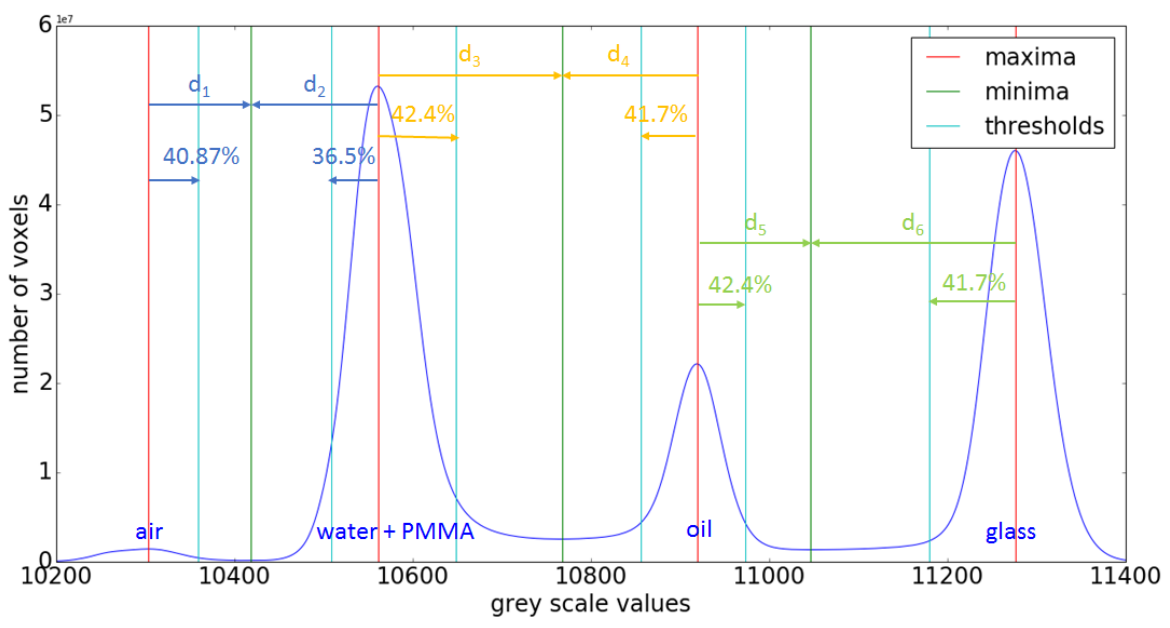

(b) microgravity thresholds

Fig. S5 Choice of thresholds for Earth-gravity experiments (a) and microgravity experiments (b). The blue graph is the intensity histogram measured over the entire volume of the tomogram. Maxima are marked with a red line, minima are marked with a green line and thresholds are marked with a turquoise line. Distances between maxima and minima are marked with arrows labelled $d_{1}$ to $d_{6}$, where orange/blue/green arrows belong to the first/second/third segmentation. Arrows between maxima and thresholds are marked with the percentage of the total distance between maximum and minimum $d_{i}$. These percentages were computed from a first segmentation and then used to choose thresholds accordingly for all other segmentations.

\begin{tabular}{|c|c|c|c|}
\hline sample & solid [voxels] & water [voxels] & air [voxels] \\
\hline PottingSoil & - & $\begin{array}{l}10000 \quad \text { (water } \\
\text { +soil) }\end{array}$ & 50000 \\
\hline
\end{tabular}

Table S3 Thresholds for the Isolated Cluster filter for potting soil data set. 


\begin{tabular}{l|l|l|l|l} 
sample & glass [voxels] & oil [voxels] & $\begin{array}{l}\text { PMMA + wa- } \\
\text { ter [voxels] }\end{array}$ & air [voxels] \\
\hline Linear RandomPack & - & 2000 & 10000 & N/A \\
\hline Quadrants & - & 2000 & 500 & 5000 \\
\hline Layers & - & 2000 & 150 & 1000
\end{tabular}

Table S4 Thresholds for the Isolated Cluster filter for microgravity data sets. 